\title{
REFLEXION ÜBER ETHNOMATHEMATIK ALS PÄDAGOGISCHE MÖGLICHKEIT
}

ORIGINAL-ARTIKEL

SCHWANTES, Vilson ${ }^{1}$

XAVIER, Márcio Pizzete ${ }^{2}$

SCHWANTES, Eloísa Bernardete Finkler ${ }^{3}$

SCHWANTES, Daniel ${ }^{4}$

${ }^{1}$ Master in Didaktik der Naturwissenschaften - Mathematik, UNIJUI - RS. Abschluss und Spezialisierung in Naturwissenschaften und Mathematik. Prof. Assistent des CCA - Zentrums für Agrarwissenschaften, Campus von Marschall Cândido Rondon, UNIOESTE, PR - Brasilien.

${ }^{2}$ Master in Didaktik der Naturwissenschaften - Mathematik, UNIJUI - RS. Abschluss und Spezialisierung in Naturwissenschaften und Mathematik. Prof. Assistent des CCA - Zentrums für Agrarwissenschaften, Campus von Marschall Cândido Rondon, UNIOESTE, PR - Brasilien.

${ }^{3}$ Spezialisierung im naturwissenschaftlichen Unterricht - Mathematik, Physik und Chemie. UNIOESTE - Staatliche Universität von Western Paraná. Abschluss: Naturwissenschaften und Mathematik. UNIJUI, RS. Professor der staatlichen Schule von Paraná.

${ }^{4}$ Er ist Professor für Pflanzenschutz und menschliche Gesundheit an der Päpstlichen Katholischen Universität von Chile, Abteilung für Pflanzenwissenschaften. Seine interdisziplinäre Position teilen sich die Fakultät für Agronomie und Ingeniería Forestal (FAIF), die Fakultät für Medizin und die Fakultät für Chemie. Forschungsprofessor in der Arbeitsgruppe Boden und Umwelt (GESOMA - UNIOESTE). Master in Agronomie von UNIOESTE, PhD in Agronomie von UNIOESTE (2013-2016) - Sandwich-Periode 
JUNIOR, Affonso Celso Gonçalves ${ }^{5}$

KRACKE, Elisa ${ }^{6}$

JUNIOR, Élio Conradi ${ }^{7}$

SCHWANTES, Vilson. Et al. Reflexion über Ethnomathematik als pädagogische Möglichkeit. Multidisziplinäre Kern Fachzeitschrift des Wissens. 04-Jahr, Ed. 07, Vol. 11, S. 148-165. Juli 2019. ISSN: 2448-0959

\section{ZUSAMMENFASSUNG}

Dieser Artikel reflektiert ethnomathematics als eine Tendenz des Lehrens, die den kulturellen Charakter des mathematischen Lernens, den sozialen Kontext, in dem sich dieses Wissen entwickelt, die Rolle des Lehrers im breiteren Kontext menschlicher Beziehungen berücksichtigt, indem er Forscher und Mediator im Bewusstsein der pädagogischen Praxis. Die Forschung berücksichtigt die Bedeutung der Geschichte

(CAPES-Stipendium) von der Universität Lissabon, am Instituto Superior de Agronomia (ULisboa).

${ }^{5}$ Research Productivity Level $1 \mathrm{C}$ von CNPq im Bereich Umweltwissenschaften mit drei Postdocs, UEM-PR (Brasilien), Universität Santiago de Compostela (Spanien), UFGGO (Brasilien). Derzeit ist er außerordentlicher Professor an der UNIOESTE-PR und Professor und Forscher am Zentrum für Agrarwissenschaften, wo er Chemie lehrt. Dozent im Masterstudiengang Agrarwissenschaften an der UEM. Derzeit ist er Ad-hocBerater bei CNPq, CAPES und Fundação Araucária. Arbeitet als freiwilliger Umweltberater bei MP-SP und CONAMA-DF.

${ }^{6}$ Bachelor in Agronomie - UNIOESTE - Staatliche Universität von Western Paraná Absolvent der Verwaltung - Unip - Universidade Paulista.

${ }^{7}$ Masterstudent in Agronomie (Pflanzenproduktion) an der Staatlichen Universität von West-Paraná (UNIOESTE). Agraringenieur, Absolvent der UNIOESTE (2014-2018), arbeitet als Forscher in der Arbeitsgruppe für Boden und Umwelt (GESOMA UNIOESTE). 
des mathematischen Wissens, seine vielfältigen Beziehungen $z u$ anderen Fachgebieten und seine Wechselbeziehung mit täglichen Aktivitäten. Der Text reflektiert auch die Möglichkeiten und Herausforderungen der Schule, pädagogische Aktionen zu fördern, die zwischenmenschliche Beziehungen im Prozess der (Re)Konstruktion von Wissen berücksichtigen. Sie winkt zu einer investigativen kritischen pädagogischen Arbeit, die in artikulierter Weise versucht, Verbindungen zwischen Alltagspraxis und Schulmathematik herzustellen.

Schlagworte: Ethnomathematik, Sozialwissen, mathematische Fähigkeiten, tägliche Aktivitäten, pädagogische Praxis.

\section{EINFÜHRUNG}

Im pädagogischen Alltag reflektieren die Pädagogen weiterhin lehrkundige Methoden und Lernmethoden. Eine der größten Herausforderungen der Schule trägt weiterhin zur Bildung kritischer, bewusster und aktiver Bürger in der Gesellschaft bei. Eine komplexe Aufgabe, bei der Schul- und Bildungsfachleute über Lehrplan, Inhalte und Bildungsprogramme hinausgehen müssen.

Die Welt, in der wir leben, obwohl wir sie nicht erkennen, hat immer grundlegend von der Mathematik abhängig gemacht. Sie war und ist immer in praktisch allem präsent, was uns umgibt. Seine Anwendbarkeit ist auch in anderen Wissenschaften relevant, wie D'ambrsio bestätigt (1996, S. 31). "Die Tendenz aller Wissenschaften ist zunehmend matematizarem wegen der Entwicklung von mathematischen Modellen, die Naturphänomene in geeigneter Weise entwickeln."

Wie wir sehen, ist Mathematik im gesamten sozialen Umfeld des Menschen imprägniert und wird durch jedes neue Lernen belegt. Alle aktuellen Berufe eignen sich aus mathematischen Kenntnissen, um Regeln, Formeln und Verhaltensweisen zu schaffen, zu pflegen und aufrechtzuerhalten. Der Maurer, die Näherin, der Koch, der Ingenieur, Techniker aller Berufe und Fachleute aus allen Bereichen der menschlichen Tätigkeit dominieren einige mathematische Kenntnisse, aber haben nicht immer die Berechnungen gelernt, um ihre Probleme in den Banken zu lösen schule. 
Für Rosa Neto (2002) besteht eine signifikante Beziehung zwischen Mathematik und Kultur, sowohl als Ergebnis unserer Anpassung an unsere Überlebensbedürfnisse im Laufe der Zeit, sondern sowohl als empirische, systematische als auch wissenschaftlich.

Ethnomathematik im Schulraum zu arbeiten, ist nach D'ambrsio (2008), zu den neuen Generationen beizutragen, um eine viel kulturelleMathematik zu kennen und zu erkennen, die mit dem täglichen Leben mehrerer ethnischer Gruppen verbunden ist. Für D'ambrsio und Rosa (2016) ist dies eine didaktische Haltung, die eine Verbesserung des Lehr-Lernprozesses der Disziplin anstrebt, indem sie in den mathematischen Lehrplan des Wissens aufgenommen wird, das sich aus dem Leben der Schüler und menschlichen Werte ergibt, wie z. B. die Zusammenarbeit, Solidarität und Ethik. Es sind Wissen, das Wertschätzung und Respekt für die verschiedenen Wege fördert, mit denen die Menschheit täglich die Problemsituationen im Altag erklärt, versteht, versteht und umgeht.

Mit diesem Bewusstsein für die Bedeutung und Rolle der Mathematik in der Entwicklung der Bürger und der Gesellschaft, versucht dieser Artikel, neben der Reflexion, Ethnomathematik zu verbreiten, in dem Glauben, dass es als pädagogischer Vorschlag kann in hohem Maße dazu beitragen, Mildern Sie die Lernschwierigkeiten und das mangelnde Interesse vieler Studenten am Studium der Disziplin.

Nach unserem Verständnis, eine pädagogische Arbeit, die auf diese Perspektive zentriert ist, macht den Schüler sicherer von seiner eigenen Fähigkeit, mathematisches Wissen aufzubauen, in der Suche nach Lösungen für die Probleme, die er in seinem täglichen Leben begegnet. Für D'ambrsio (1996) ist es jedoch notwendig, dass der Lehrer eine neue Haltung im Klassenzimmer einnimmt, ein neues Paradigma sucht, das das bereits abgenutzte Lehren-Lernen ersetzt, das auf einem veralteten Verhältnis von Ursache-Wirkung basiert.

Pompey und Monteiro (2001, S. 14), warnen, 
Der Paradigmenwechsel ist jedoch ein komplexer Prozess; Es ist notwendig, sich ändern zu wollen und zu glauben, dass dies möglich ist. Mehr als zu finden, dass wir uns ändern müssen, ist es notwendig, die Überzeugung zu haben, dass es immer eine neue Art des Lehrens gibt, dass es immer möglich ist, sich zu ändern.

Die Autoren betonen, dass die pädagogische Arbeit in dieser Perspektive den Lehrer erfordert, den Umfang ihrer Rolle zu verstehen und zu klären, sowie die Bedeutung der mathematischen Inhalte, mit denen sie mit den Schülern arbeiten. Theoretisch ist es notwendig, den Studenten auf eine ganzheitliche Weise zu verstehen, um ihre Entwicklungsbedürfnisse auf intellektueller, physischer, emotionaler, sozialer und kultureller Ebene zu identifizieren. Sie wiesen daher darauf hin, dass

Die derzeitigen Lehrer stehen vor einer großen Herausforderung: Sie werden in einem fragmentierten Prozess ausgebildet die Grenzen überwinden müssen, die ihnen diese Formation auferlegt, und die Grenzen der Inhalte, die teilweise und a-historisch gesehen werden, extrapolieren müssen (POMPEU und MONTEIRO, 2001, S. 15).

Die Aufgabe des Lehrens und Lernens in diesem Ansatz unterscheidet sich in der Tat von der traditionell bekannten. Es ist Teil dessen, was wir für einvernehmlich halten, das heißt, dass alle Kulturen und Völker ihre eigenen Wege entwickelt haben, ihre eigene Realität zu erklären und zu verändern. Dieses Wissen und die Gegenwart in jedem Prozess der menschlichen Entwicklung, die jeder Kultur angemessen sind, sind natürlich im Prozess, in der Phase der Evolution und des ständigen Wachstums, und tragen so zur Ausarbeitung von systeted Knowledge bei.

D'ambrsio (1993) betont, dass die Menschen eine Kultur haben, sich [etno]mit ihrer eigenen Kultur auseinandersetzen [matema]und sie erklären, aber jeder in seiner eigenen Art und For[tica]m. Dieser Befund führte zu dem Namen Ethnomathematik, der wiederum die Erklärungen der Gemeinschaften berücksichtigt und sie mit den universellen Formen des Wissens tropft. Es ist eine Tatsache, dass jedes Kind vor der 
Ankunft in der Schule weiß, wie man perfekt mit seiner Realität umgeht, die sich ständig weiterentwickelt.

Nach Ansicht des Autors ist Ethnomathematik Teil des Prinzips und der Idee, die Mithänge der Tradition und Kultur der Kinder zu integrieren und sie als gültig für die Erfüllung ihrer Zwecke anzuerkennen. So verwirrt ethnomathematics mit der Neugier des Kindes, wendet sich zuerst der Umgebung zu, in der sie lebt, und erfüllt eine Aufgabe, die die Schule vergessen hat: die Kreativität der Schüler durch Projekte zu befreien und zu entwickeln. investigativ.

Diese pädagogische Perspektive ist die sogenannte Ethnomathematik, eine Tend ${ }^{[8]}$ enz des Mathematikunterrichts, die in ihrer pädagogischen Praxis die sozialen und kulturellen Aspekte der Menschheit berücksichtigt. Sie wird als lehrische Tendenz charakterisiert, die in die Diskussion eingeht und versucht, über die verschiedenen Formen von Matematizar nachzudenken, die in unserer soziokulturellen Realität vorhanden sind.

Es schlägt eine kritische Ausbildung vor, deren Grundlage in den Studien und Schriften von Ubiratan D'ambr'sio unterstütz ${ }^{[9]}$ t wird, die auf einen methodischen Vorschlag der mathematischen Bildung verweisen, der die Erfahrungen der Studenten schätzt. Eine Vision mit Merkmalen, die die Prozesse durchdringen: anthropologisch, sozial und politisch, die die lokale Kultur jeder gesellschaftlichen Gruppe in die Diskussion bringen, als eine menschliche Aktivität, die kulturell durch den Kontext bestimmt wird, in dem sie durchgeführt wird.

In diesem Zusammenhang wurde die Ethnomathematik in den letzten Jahrzehnten national und international als didaktisch-pädagogischer Vorschlag für den Unterricht der Mathematikdisziplin geweiht. Nach D'ambrsio (2001) "Ethnomathematik ist die Mathematik von kulturellen Gruppen praktiziert, wie städtische und ländliche Gemeinschaften, Gruppen von Arbeitnehmern, Berufsklassen, Kinder einer bestimmten Altersgruppe, indigene Gesellschaften, und so viele andere Gruppen, die durch die gemeinsamen Ziele und Traditionen der Gruppen identifiziert werden "(S. 9). 
Es ist, so der Autor, eine Tendenz des Lehrens, wo Wissen sich ganzheitlich manifestiert, das heißt, es ermöglicht den verschiedenen kulturellen Gruppen, ihre Welt zu kennen, zu verstehen und zu erklären, mit Mathematik, Entwicklung Fähigkeit, durch sie in ihrer Realität zu verwalten und sich einzumischen. Sie winkt der Möglichkeit, einen Schullehrplan zu erstellen, der das soziokulturelle Leben der Schüler in pädagogische Praktiken als eine der Formen der sozialen Eingliederung einbezieht.

In den Worten von D'ambr'sio (1993): "Ethnomathematics ist ein Programm, das darauf abzielt, die Prozesse der Generierung, Organisation und Weitergabe von Wissen in verschiedenen kulturellen Systemen und die interaktiven Kräfte, die in und zwischen den drei Prozessen wirken, zu erklären" (S. 7).

In dieser Lehrtendenz ist der Lehrplan dynamisch, kontextualisiert, erkennt die kulturelle und soziale Pluralität der Studierenden, ermöglicht es dem Schüler, ein aktives Fach zu werden, partizipativ im Prozess der Transformation seines lokalen, regionalen und globalen Umfelds.

In Bezug auf das Ethnomathematik-Programm denkt D'ambrsio (2001, S. 17) auch darüber nach, dass

Der große Motivator des Forschungsprogramms, das ich Ethnomathematik nenne, ist zu versuchen, das mathematische Knowhow in der Geschichte der Menschheit zu verstehen, kontextualisiert in verschiedenen Gruppen von Interesse, Gemeinschaften, Völker n. Chr. und Nationen.

Pompey und Monteiro (2001), in Bezug auf die Annahme einer pädagogischen Praxis, die das Wissen/ tun in den Unterricht bringt, argumentiert, dass er

Sie ist durchdrungen von Gewissheiten, die nicht von der Wissenschaft "gegeben" werden, sondern von Werten, Traditionen und Respekt für diejenigen, die lehren. So hat es eine "emotionale Gewissheit" durch 
Erfahrung bestätigt, anders als die "wissenschaftliche Gewissheit", dass im Allgemeinen anfällig für rationale Demonstration und Mathematik, obwohl, wenn gelehrt, auch die Einführung emotionaler Werte, in der Person der Lehrer (S. 134 - 135).

In der pädagogischen Aktion ist die Beziehung zwischen den Lern- und Lernprozessen dialogisch, denn es ist der Kontext in diesen Interaktionen, der Lernmöglichkeiten begünstigt. In dieser Dynamik bedeutet Ethnomathematik und Wissen/Tun ins Klassenzimmer, die Erfahrungen und das Wissen, die in den täglichen Aktivitäten der verschiedenen Arbeiterklassen vorhanden sind, wiederzuerlangen, um den Wissenschaftlichkeitsismus des Wissens zu überwinden. akademisch.

Pompey und Monteiro (2001) wiesen jedoch in Bezug auf die Arbeit im Klassenzimmer das formale und informelle Wissen darauf hin, dass

[...] Es ist notwendig, dass eine Gruppe die Grenzen ihres Wissens kennt und anerkennt, um darüber hinauszugehen und das Neue mit Bewusstsein zu suchen, das die Bedeutung und Gültigkeit dieses neuen zu kennen und zu diskutieren. Nur so wird er wählen können, wann und welches Wissen verwendet werden soll (S. 53-54).

In den Schulen im Allgemeinen wird es gelehrt und gelernt nur Mathematik diktiert als offizielle, während in alltäglichen Aktiv[comercias, agrícolas, de prestação de serviços, entre outras]itäten, sie anwenden und verwenden mathematisches Wissen nicht offiziell anerkannt, sondern sozial konstruiert, validiert und Von der Gesellschaft geschätzt.

Kurz gesagt, manchmal scheint es, dass die Bürger mit der Existenz von zwei Mathematik leben: die Schule, in [oficial]der Schule gelebt, theoretisch, abstrakt und selektiv und die des realen Lebens, die die aufkommenden Bedürfnisse der Bürger erfüllt. Es wird wahrgenommen, dass diese "andere Mathematik", selten von der Schule akzeptiert, zeigt sich praktisch, dynamisch und abgestimmt mit allen Bereichen der Gesellschaft, weit verbreitet in den Routineaktivitäten der Bürger verwendet. 
Nach D'ambrsio (2001), Ethnomathematik, zusätzlich zu nicht leugnen formale Mathematik, versucht, zur Diskussion jede Form von Matematizar zu bringen, analyse argumentativ und reflexiv jeden Kontext, in dem Mathematik eingefügt wird. Sie versteht und diskutiert die verschiedenen Formen von Matematizar, vorausgesetzt, dass die Ziele jedes Kontextes erfüllt werden, jeder Gruppe von Gleichen in ihren spezifischen Situationen.

Daher erfordert die Arbeit mathematik in einem Ethno- ${ }^{[10]}$ Ansatz, wie D'ambrsio (1993) vorschlägt, dass der Pädagoge lernt, mit den verschiedenen kulturell aufgebauten Kenntnissen umzugehen und unter den Schülern zirkulieren. Sie schlägt vor, dass die Schule ihre Praxis im Einklang mit den Wünschen und täglichen Bedürfnissen der Menschen überdenkt und die soziale Funktion des mathematischen Wissens rettet.

In dieser Dynamik muss der Pädagoge offen sein für Veränderungen in der Art und Weise des Handelns und Arbeitens. Sie müssen sich die Erfahrungen anhören, die die Schüler von zu Hause, aus ihrer Gemeinschaft, aus den Beziehungen zwischen ihnen mitbringen, die aus diesem dialogisch-interaktiven Prozess suchen, um den mathematischen Inhalt zu bearbeiten. Und neben der Einbeziehung neuer Methoden in ihre Praxis, die ständige Analyse und Bewertung ihrer Praxis, Verbesserung der pädagogischen Maßnahmen zuvor erlebt. Schließlich muss sie das mathematische Wissen aus den persönlichen Erfahrungen der am Bildungsprozess Beteiligten konstruieren.

So schlägt die Praxis der Ethnomathematik im Klassenzimmer, so Professor Ubiratan D'ambrrésio, vor, einen viel breiteren Weg der Geselligkeit und des Austauschs von unterschiedlichem Wissen zu gehen.

Durch die Praxis Ethnomathematik, wird der Pädagoge die großen Ziele der mathematischen Bildung $\mathrm{zu}$ erreichen, mit unterschiedlichen Perspektiven für verschiedene kulturelle Umgebungen und Produktionssysteme. Es ist gerechtfertigt, den Studenten in den Produktionsprozess seiner Gemeinschaft und sozialen Gruppe 
einzufügen und die kulturelle und historische Vielfalt in verschiedenen Kontexten zu belegen (D'ambr'sio, 2008, S. 8).

\section{RECHTFERTIGUNG}

In den Schulen wird in einem beträchtlichen Prozentsatz wahrgenommen, dass der Lehr-Lern-Prozess der Mathematik weiterhin unveränderlich ist, das heißt, der Lehrer bringt die Informationen weiterhin bereit, der Schüler empfängt sie passiv, transparent, in diesen Fällen, Ein akzentuierter Konformismus und historischer Determinismus. In der Praxis gibt es auch eine unzusammenhängende Methodik zwischen dem, was in der Schule gelernt wird, und dem, was die Gesellschaft wirklich von ihren Bürgern verlangt.

Laut Santos (2016):

Der Mangel an Kontextualisierung in der Lehre der Mathematik kann die Entmutigung der Disziplin verursachen, wiederauferstehen die traditionellen Methoden, die Mathematik als eine Wissenschaft, die alle Dinge bereit gebracht, als ob es ein fertiges Wissen und über. (p. 5).

Eine Lehre, die den Lernenden zu einem mechanischen Lernen führt. Darüber hinaus gibt es immer noch diejenigen, die glauben, dass mathematisches Wissen nur für Privilegierte möglich ist, nicht für alle zugänglich, was nach Ubiratan D'ambrsio mit dem System der "Kaste" vergleichbar ist, das auf einige Länder angewendet wird.

[...] Einige mathematische Pädagogen sehen Mathematik als eine privilegierte Form des Wissens, zugänglich nur für einige besonders begabte, und deren Lehre sollte strukturiert werden, unter Berücksichtigung, dass nur bestimmte Köpfe, in irgendeiner "besonderen" Weise, kann Assimilieren und schätzen Die Mathematik in ihrer Füll[...]e. (D’AMBRÓSIO, 1996 p.9). 
In dem Versuch, diese Situation zu minimieren und Motivation und Freude in den Unterricht zu bringen, suchen wir durch diese bibliographische Forschung, sowohl eine kontextualisierte Mathematik für die Schüler, als auch einen methodischen Weg, der bessere Ergebnisse liefert . Eine Lehralternative, die mehr Signifikation für die Inhalte der Mathematik studiert ermöglicht. Etwas Motivierendes, Attraktives, das dem Lernenden wirklich in einer Weise dient, die es inm ermöglicht, Situationen-Problem in einer kontextualisierten Weise zu lösen, abzuleiten und Entscheidungen im Angesicht seiner Realität zu treffen, zu verstehen, dass, einschließlich der formalen Mathematik lebendig ist, vorhanden ist In unserem Alltag.

Eine Mathematik, die in der Schule gelehrt wird, die in der Realität des Lebens der Schüler vorhanden ist, die Interaktion zwischen Schule und Gemeinschaft sucht, in der Möglichkeit, ein Werkzeug zur Transformation des sozialen Umfelds zu sein. Ein Wissen, dessen Konstruktion und Verständnis nicht nur für Mathematiker, Wissenschaftler, Meister oder Ärzte möglich ist, sondern auch für gesellschaftliche Gruppen, die in ihrer Arbeitstätigkeit Fähigkeiten entwickeln, berechnen, zeichnen, erstellen oder Spielen Sie, nach Ihren Interessen und Bedürfnissen.

So können die Schüler durch das erworbene Wissen zu singulären und reflexiven, emanzipierten, selbstbewussten und interaktiven Fächern in der Gemeinschaft werden. Giardinetto (1999) denkt in seinen Schriften darüber nach, dass das Thema nur ein mitwirkender Bürger seiner Gesellschaft werden wird, wenn er sich "von kulturellen Instrumenten aneignet" (S. 73).

Wir wissen und stimmen zu, dass Mathematik eine Wissenschaft ist, die sowohl im täglichen Leben der Bürger als auch in Forschungszentren präsent ist. Angesichts dieses Umfangs können wir daher die Lehre dieser Disziplin nicht auf ein einfaches Auswendiglernen von Regeln, Techniken und der formalen Kenntnis von Definitionen beschränken, denn "... Lehre ist nicht der Transfer von Wissen, sondern die Schaffung von Möglichkeiten für die eigene Produktion oder ihre Konstruktion. " (FREIRE, 1996, S. 52). 
Mathematik aus der Perspektive der Ethnomathematik zu lehren, wird unmöglich, ohne das Wissen und die Werte zu retten, die die Schüler von zu Hause mitbringen. Damit dieser Akt verwirklicht werden kann, ist es für den Lehrer unerlässlich, seinen Schülern zuzuhören, wie es für den Schüler, der mit seinem Lehrer und seinen Kollegen spricht, unerlässlich ist.

Laut Dem Pädagogen Paulo Freire

"Der Aufbau dialogischer Beziehungen unter den Grundlagen der universellen Ethik des Menschen, wie eine spezifische menschliche Praxis, impliziert das Bewusstsein der Menschen, so dass sie sich tatsächlich in den historischen Prozess als Subjekte einfügen können, die ihre Eigene Geschichte. " (FREIRE, 1996, p10.)

Der mathematische Pädagoge Paulus Gerdes, in seinen verschiedenen Publikationen nimmt immer einen Diskurs, der die mathematischen Traditionen der kulturellen Gruppen rettet, dass vor der Kolonisierung wurden nach inm "versteckt" und durch eurozentrische Konventionen in auferlegt Gesellschaft, sowie im Schulraum.

In dieser Perspektive (GERDES, 2010, S. 142)

Ethnomathematik ist der Forschungsbereich, der die vielfältigen Zusammenhänge und Zusammenhänge zwischen mathematischen Ideen und anderen kulturellen Elementen und Bestandteilen wie Sprache, Kunst, Handwerk, Bau wesen und Bildung untersucht. Es ist der Forschungsbereich, der den Einfluss kultureller Faktoren auf das Lehren und Lernen von Mathematik untersucht.

Laut dem Autor (2010, S. 142-143) "Ethnomathematik zeigt, dass mathematische Ideen in allen menschlichen Kulturen existieren, in den Erfahrungen aller Völker, aller sozialen und kulturellen Gruppen, sowohl von Männern als auch von Frauen." In dieser kulturellen Perspektive zeigt sich auch, dass 
[...] Alle Völker aller Zeiten können zu dieser universellen Mathematik beitragen. Alle Völker haben das Recht, das angesammelte Wissen zu lernen und zu genießen und zu ihrer Bereicherung beitragen zu können. Hier liegt eine ethische und moralische Dimension der ethnomathematics Reflexion. (GERDES, 2010, p.144)

Mehrere Autoren haben bereits berichtet und durch unsere Berufserfahrung haben wir auch festgestellt, dass die verschiedenen Formen von Matematizar so präsent im täglichen Leben der Bürger sind, dass viele Menschen sie in ihren täglichen Aufgaben nutzen, obwohl sie nie besuchte eine Schule. Dieses Wissen, das manchmal empirisch erworben wird, ist von außerordentlichem Wert und Reichtum, so bedeutend, dass viele nicht immer die Sensibilität haben, sie wahrzunehmen. Wir teilten mit Pompey und Monteiro (2001), als sie postulieren, dass,

"Der Mensch bezieht sich auf die Welt um inn herum, versteht dies als Weltleben, das heißt, die Welt, in der die Menschen sind, die uns durch Einflüsse erzieht, die uns durch unsere Beziehungen zu ihm erreichen" (S. 21).

In dieser pädagogischen Perspektive ist es jedoch nicht beabsichtigt, eine neue Mathematik vorzuschlagen, sondern über die Möglichkeit nachzudenken, in den verschiedenen kulturellen Gruppen ihre Art von Matematizar zu identifizieren, ihre Berechnungsmethoden zu bewerten und zu prüfen. Kennen, erklären und verstehen Sie die Welt, die sie umgibt, und bereichern Sie sie in dieser dynamischen Schulmathematik. Wie D'ambrosius (2001) berichtet, ist es eine Mathematik, die "... Es ist Teil des täglichen ", des Know-hows (S. 25). In der Tat ein pädagogischer Vorschlag, der in integrierter Weise das formale und informelle Wissen funktioniert.

Wir glauben, dass von dem Moment an, in dem wir Ethnomathematik als eine pädagogische Alternative betrachten, die Mathematik in verschiedenen sozialen, kulturellen und historischen Umgebungen betrachtet, neue Methoden in den Unterricht integriert werden. Es wird die Schulmathematik zu einem Instrument, um die 
Entwicklung und Produktion des in diesen verschiedenen Kontexten erzeugten Wissens zu erklären und zu verstehen und die Schüler zu motivieren.

Das Wissen zu verstehen, das innerhalb einer bestimmten Gemeinschaft entwickelt wird, bedeutet, Ethnomathematik in diesem Umfeld zu verstehen, das heißt, auf die verschiedenen Formen der Manifestation von Wissen zurückzublicken, nicht nur des mathematischen Wissens, sondern Das Wissen über das Leben, die Organisation, die Konstruktion und Strukturierung dieses Wissens und die Herstellung, dass sie die Geburt dieser Menschen sind.

Jedoch, viele Mathematik-Pädagogen, vielleicht aufgrund ihrer universitären Ausbildung auf der Technicist Linie zentriert, zeigen Schwierigkeiten, mit dem alten Paradigma zu brechen und die Annahme einer Lehrmethodik, die Schulmathematik mit der Mathematik der leben. Diese Tatsache wird zu einem Hindernis für die Umwandlung des Klassenzimmers in einen Raum der Diskussion und kritischen Analyse soziokulturell aufgebauten Wissens. Für Pompeius und Monteiro (2001, S. 19) ist es notwendig, nach Wegen zu suchen, bestehende Konflikte zu überwinden, denn "wir leben einen Moment, in dem die Fragmentierung des Wissens das Verständnis der Realität einschränkt".

Wir müssen darauf vertrauen können, dass das, was die Schüler in der Schule tun und lernen, stärker auf die Handlungen und Bedürfnisse der verschiedenen Bereiche menschlicher Aktivitäten außerhalb der Schulmauern abgestimmt werden kann. Der Pädagoge integriert und bringt diese sozial aufgebauten Kenntnisse einfach ein und bringt sie ins Klassenzimmer ein. Wir teilen an dieser Forschung mit den Schriften von D'Ambrosio und Rosa, da sie Ethnomathematik als ein Forschungsprogramm in Geschichte und Philosophie der Mathematik betrachten, mit pädagogischen Implikationen,

Dieses Forschungsprogramm stellt also eine Forschungsmethodik dar, die darauf abzielt, lokale mathematische Praktiken zu analysieren, da es versucht, mathematisches Wissen (Ideen, Begriffe, Verfahren, Verfahren 
und Praktiken), die Ursprung in verschiedenen kulturellen Kontexten im Laufe der Geschichte (D'ambr'sio und ROSA, 2016, S. 17).

Eine pädagogische Arbeit, die sich auf diese Perspektive konzentriert, schätzt den Menschen und sein informelles Wissen, das in und durch die Kultur, in der er lebt, konstituiert ist, kann der Beginn einer Veränderung in der Lehre dieser Disziplin sein. Laut D'ambrsio (2001) trägt eine Lehre, die "Praktiken enthüllt, die außerhalb des Schulumfelds ergriffen [pode]wurden, zu einem kritischen Blick auf die Realität bei" (S. 23).

Die Schule als eine Institution, die die Fortsetzung der Familie in ihrem breiteren Kontext darstellt, muss verstehen, dass der gesamte Lehr-Lern-Prozess, der Teil der früheren Erfahrungen der Bürger, der Formen von Matematizar von Schülern außerhalb der Schule, bereichert das Bildungsakt. In den Worten von Giardinetto (1999) "Das tägliche Leben selbst braucht Eingriffe des Nicht-Quotidian" (S. 7).

In diesem Sinne würde es verlassen, um die offizielle Mathematik als die einzige Mathematik zu arbeiten, die Aufmerksamkeit verdient. Wir würden die Erfahrungen und Kenntnisse der Schüler vor der Schulphase berücksichtigen und in die Diskussion einbringen. Die Schule, die all diese Erkenntnisse dialogisch zusammenstellt, verwandelt das Klassenzimmer in ein permanentes Lernlabor.

In diesem Trend ist eine der Rollen der Pädagogen, den natürlichen Wunsch der Schüler zu stimulieren, die verschiedenen Mathematik in den verschiedenen kulturellen Gruppen zu untersuchen. Durch das Verständnis der Prozesse von Matematizar jeder Kultur, vergleichen sie dieses Wissen mit formalem Wissen, Systate und validiert von der Schule, mit der Möglichkeit, ihr eigenes Denken zu organisieren, durch diese Reflexion die kulturelle Bedeutung und sozial jedes mathematischen Wissens, das in der Welt des Lebens zirkuliert. In den Worten von D'ambrsio (2001) könnte man besser verstehen, dass "das Verhalten jedes Einzelnen, verbunden mit seinem Wissen, durch die Anwesenheit des anderen verändert wird" (S. 32). 
Nach D'ambrsio (1993) bedeutet Mathematikunterricht in dieser Konzeption, das zu respektieren, was der Schüler bereits weiß, besteht darin, das historisch konstruierte Wissen in Kulturen zu erkennen, dialogische Momente kritischer Fragen zu etablieren, die sich erneuern und Das Vorwissen aller Teilnehmer am Lernprozess.

Für D'ambrsio (2001) Es respektiert und wertet jede der bestehenden Mathematik, und "... Es ist die Kommunikation, die es ermöglicht, Strategien für gemeinsames Handeln zu definieren "(S. 58). Die Schule erklärt durch dialektische ${ }^{[11]}$ Reflexion die Produktionsweisen mathematischer Bedeutungen, die außerhalb ihres spezifischen Kontextes erzeugt werden. In diesem Ansatz ist es, als ob die Schulmathematik mit den Formen des Matematizar der umgebenden Welt verbunden, anstatt sie zu ersetzen. Während die Schulmathematik diese Vielfalt des Wissens ignoriert oder nicht diskutiert, die in der Kultur jedes Volkes produziert wird, wertet ethnomathematics wertet und bringt diese Unterschiede in die Debatte, indem sie ihre mathematischen Modelle und das damit verbundene Wissen erkennt. .

Nach D'ambrsio (2001), während traditionelle Mathematik und ihre Lehre versuchen, die Konzepte zu universalisieren, machen sie immer abstrakter und verallgemeinern, Ethnomathematik versucht, sie neu zu ordnen, um sie spezifischer und anwendbarer auf Kontext der gelebten Realität. Zusammenfassend wird eine kohärentere Art des Gehens vorgeschlagen. Es versucht, Mathematik von Büchern zum[da escola] Leben und Leben in die Schule zu bringen, erkennend, dass beide eine Geschichte und Bedeutung für das Verständnis der Realität in all ihren Dimensionen haben.

Die Grundidee dieser Tendenz des Mathematikunterrichts, so D'ambrsio (2001), besteht darin, die Erfahrungen von Studenten, Verwandten, Gruppen von Gleichen, verschiedenen Kulturen und allen Formen des Selbstmatematizaren jedes Volkes zu berücksichtigen. Es handelt sich um Wissen, das durch die praktische Notwendigkeit zur Lösung spezifischer Probleme von Gerechten, Kleinbauern, Maurern, Zimmerleuten, indigenen Gruppen, Siedlern usw. hergestellt wird. Es handelt sich um unterschiedliche Kenntnisse, die von verschiedenen Gruppen erstellt werden, um Problemsituationen aufzuklären. 
Für Pompey und Monteiro (2001) zeichnet sich eine pädagogische Praxis aus, die sich gleichzeitig der Zersplitterung des Wissens widersetzt, durch die kulturelle Verstellung des historischen Wissens und die Opportunisierung der Menschen zur Rettung ihrer kritischen reflektierenden dialogischen Fähigkeit. Sie versteht, so die Autoren, den Menschen in seiner Gesamtheit und das Wissen in seiner ganzen Komplexität.

In den Worten von Pompeius und Monteiro (2001), eine Lehre, die "Den Menschen und nicht das Kapital als zentralen Wert der sozialen Beziehungen" (S. 26) setzt. Laut D'ambrsio (2001) erfordert eine Arbeit, die sich auf diese Perspektive konzentriert, von Denkern eine gute Dosis Humanismus, denn "der Schüler ist wichtiger als Programme und Inhalte" (S. 86).

Mathematik arbeitete an einem Ethno-Ansatz bringt in den Unterricht Erfahrungen, die Kommunizität und argumentativen Dialog erfordern. Diese didaktische Haltung wiederum führt die am Bildungsprozess Beteiligten zu einem persönlichen und kollektiven Wachstum, das durch die permanente Reflexion beider und beider Praktiken ermöglicht wird.

Gemeinsam unterrichten und lernen Lehrer und Schüler gleichzeitig, kennen die Welt, in der sie leben, kritisch und bauen Beziehungen von gegenseitigem Respekt, Gerechtigkeit auf, die ein echtes Klima der Disziplin bilden, durch dialogische Beziehungen, die das Klassenzimmer zu einer Herausforderung machen. Interessant und herausfordernd für alle Beteiligten. "Wer lehrt, lernt beim Lehren und wer lernt, lehrt im Lernen" (FREIRE, 1996, S. 38).

Giardinetto (1999): "Der Lehrer kann und sollte das tägliche Wissen als Unterstützung für den Lehr-Lern-Prozess nutzen" (S. 68), indem er den Schüler in den Bereich der Argumentation bringt und die Gewohnheit des kritischen Lesens, der Forschung, der Fragen, Kreativität, unverzichtbar für die Bürgerausbildung.

Durch den Start dieses Blicks auf ihre Praxis, die Studenten 'Vorkenntnisse geschätzt und andere gebildet werden, immer aus dem bekannten. Wichtig ist die Etablierung von Wissensbindungen der Gemeinschaft mit dem Wissen der Schule und dem 
Wissen der Schule mit dem Wissen der Gemeinschaft. Durch diese Etablierung und diese Beziehungen ist es möglich, beiden Wissen Bedeutung enden zu können. Für D'ambrsio (2001) stellt Wissen in diesem Zusammenhang einen dynamischen Charakter dar, der immer offen für neue Ansätze ist.

Für Freire (1996) Ist die Rolle des Lehrers mit der Vorstellung verbunden, dass der Unterricht nicht Wissen vermittelt, sondern Bedingungen für seinen Aufbau schafft. Es bedeutet zu erkennen, dass Schüler und Lehrer gemeinsam im Klassenzimmer lernen, da sie alle viel Wissen über die schulischen und außerschulischen Erfahrungen mitbringen, die sie während ihres Bestehens gemacht haben.

Wir glauben, dass Ethnomathematik jedem Pädagogen bei dieser pädagogischen Möglichkeit helfen kann, die Routineaktionen des "Gebens von bereiten Klassen" durch eine Haltung zu ersetzen, die das Lernen des Schülers beim Aufbau seines eigenen Wissens ,, Frucht von Interaktion zwischen Probanden in diesem dialogischen Prozess. Auch weil die Gesellschaft des Wissens, in der wir leben, heute eine ganzheitliche Haltung in der Bildung inrer Bürger fordert, die in der Lage sind, mit Dynamik in allen Kontexten zu interagieren, neue Bedeutungen für die Mathematik zu konstruieren, $z u$ erforschen Verschiedene Formen von Situationen-Problem verschiedener menschlicher Aktivitäten.

\section{TOR}

Von der Reflexion an den Mathematik-Pädagogen zu beitragen, eine investigative kritische Haltung in Bezug auf den Unterricht der Mathematik zu nehmen, aus der Perspektive der Initiierung seiner Lehrtätigkeit durch die Herstellung von Bindungen zwischen täglicher Mathematik und Schulmathematik.

\section{METHODIK}

Die theoretischen Inspirationen dieses Artikels sind auf Lesungen und Reflexionen über Mathematik-Bildung, Ethnomathematik und die interkulturelle Perspektive des Lehrens zurückzuführen. Die Schriften versuchen, über den ethnomathematischen 
Ansatz in der Perspektive zu reflektieren, dass eine Lehre, die auf den Vorkenntnissen der Schüler basiert und ihre Kultur und ihre Modi von Matematizar schätzt, ein aggregationenfaktor des Wissens sein wird, der einen humaneren Charakter verleiht. Für Disziplin.

\section{ERWARTETE BEITRÄGE}

Möge die Bedeutung der Ethnomathematik bei der Ausbildung des Studenten wahrgenommen werden, so D'ambrsio (2001), da ihre kulturelle und anthropologische Voreingenommenheit auf den Weg verweist, der zur Achtung der Unterschiede, des Verständnisses und der Inklusion führt, die für unsere Realität so notwendig sind. sozial.

Dass der Unterricht von Mathematik im Klassenzimmer aus dieser pädagogischen Perspektive einen effektiven Dialog zwischen dem mathematischen Wissen in verschiedenen Kontexten ermöglicht und dass Lehrer mehr über die kulturelle Vielfalt der Disziplin und ihre Bedeutung für die Staatliche Bildung.

Dass aus dieser Reflexion die pädagogische Praxis im Klassenzimmer auf die Entwicklung der vollen Bürgerschaft der Schüler umgestaltet werden kann. Das, nach Pinheiro und Rosa (2016), die:

[...] Mathematiklehrer tauchen in die kulturelle Dynamik der Schüler ein und nutzen Lehr- und Lernstrategien, die die kulturelle Dimension im Klassenzimmer schätzen, so dass eine inklusive mathematische Bildung entwickelt werden kann, die effektiv Beitrag zur gesellschaftlichen Transformation (S. 79).

Dass diese Bemühungen zu neuen Elementen für den Unterricht der Disziplin der Mathematik beitragen können, so dass im Klassenzimmer mehr und mehr Mathematik und Realität zu studieren, als Möglichkeiten, jeden Kontext zu kennen und zu interpretieren, in einem Prozess, in dem der Lehrer wird Ständig (re) produzieren, (re) konstruieren, (re) bedeuten Wissen und Wissen. 
Wir hoffen auch, dass die Leser die Möglichkeit wahrnehmen, in den Unterricht der Mathematik einen differenzierten Blick auf die Realität und auf die Mathematik selbst einzufügen und dass dieser Blick mit dem mathematischen Lernen des Erziehers korrodiert, um Sie zu nehmen, an Diskussionen über Mathematik innerhalb und außerhalb des Klassenzimmers.

Wir wollen, dass das Klassenzimmer, basierend auf der Lehrmethodik, beginnt, in den pädagogischen Prozess einzubinden/zu analysieren, Problemsituationen, deren Lösung sowohl formale Mathematik als auch informelle Mathematik berücksichtigt. Möge die Disziplin als menschliche Aktivität betrachtet werden, die das Interesse der Schüler noch mehr motiviert.

Wir betonen, dass es nicht darum geht, die Mathematik durch eine andere zu ersetzen, sondern darum, die verschiedenen Modi von Matematizar in verschiedenen Kontexten zu respektieren, innerhalb der Zwecke, zu denen sie vorgeschlagen werden. Daher glauben wir, dass dieser pädagogische Ansatz uns auf eine kontextualisierte Bildung verweisen kann, die bereichert und die Teilnehmer motiviert, Teil des Prozesses zu sein.

Mit den Worten von RAM one,

[...] Mathematik unterricht in dieser Konzeption wird es dem Schüler ermöglichen, die Inder-Konzepte mit ihrer täglichen Erfahrung zu verknüpfen, entsprechend ihrem natürlichen, sozialen und kulturellen Umfeld. Es geht nicht darum, die akademische Mathematik abzulehnen, sondern Werte zu berücksichtigen, die in Gruppenerfahrungen erfahren sind, wenn man die historisch-kulturellen Bindungen betrachtet (CARNEIRO, 2012, S. 3).

Schließlich beabsichtigt sie, zur Konstitution des Forschers Professor seiner Praxis beizutragen, erweckt ihn/sie, auf kontinuierliche Weise zu lernen, lehrer zu sein, entsprechend den aktuellen Bildungsbedürfnissen, das Klassenzimmer in eine 
Lernumgebung zu verwandeln. Demokratischer Wissensaustausch, Rettung historisch-sozial aufgebautes Wissen über die Menschheit.

\section{BIBLIOGRAPHISCHE HINWEISE}

CARNEIRO, K. T. A. Cultura Surda na aprendizagem matemática da sala de recurso do Instituto Felipe Smaldone: uma abordagem etnomatemática. Anais do 4ํㅡㄹ Congresso Brasileiro de Etnomatemática. Belém, PA: ICEm4, 2012.

D’AMBRÓSIO, Ubiratan. O programa Etnomatemático: Uma síntese. Acta Scientía, v.10, n.1, Jan/jun.2008.

Etnomatemática - elo entre as tradições e a modernidade. Coleção Tendências em Educação Matemática, 1. Belo Horizonte: Autêntica, 2001, 112p.

Da realidade a ação: Reflexões sobre a educação e matemática. Campinas: Unicamp, 1996.

. Etnomatemática: Arte ou técnica de explicar e conhecer. Editora Ática, Série Fundamentos, 2. edição, São Paulo, 1993.

D'AMBROSIO, U.; ROSA, M. Um diálogo com Ubiratan D'Ambrosio: uma conversa brasileira sobre etnomatemática. In BANDEIRA, F. A.; GONÇALVES, P. G. F. (Orgs.). Etnomatemáticas pelo Brasil: aspectos teóricos, ticas de matema e práticas escolares. Curitiba, PR: Editora CRV. 2016. pp. 13-37.

FREIRE, Paulo. Pedagogia da autonomia: Saberes necessário a prática educativa. São Paulo: Paz e Terra, 1996.

GERDES, Paulus. Geometria dos Trançados Bora na Amazônia Peruana, Livraria da Física, São Paulo, 2010, 190 p. São Paulo: Editora Livraria da Física, 2011, 190p. 
GIARDINETTO, José Roberto Boettger. Matemática escolar e matemática da vida cotidiana. Coleção polêmicas do nosso tempo, autores associados, Campinas - São Paulo, 1999, 128p.

MONTEIRO, A.; Pombeu, G. Jr. A matemática e os temas transversais. Editora Moderna, São Paulo, 2001, 160p.

PINHEIRO, R. C.; ROSA, M. Uma perspectiva etnomatemática para o processo de ensino e aprendizagem de alunos Surdos. RPEM, v. 5, n. 9, p. 56-83, 2016.

ROSA NETO, R. Didática da matemática, São Paulo: Ática, 2002.

SANTOS, Lijecson Souza dos. Contextualização Matemática Em Situação De Ensino E Aprendizagem No Eja. Disponível em: https://editorarealize.com.br/revistas/cintedi/trabalhos/TRABALHO_EV060_MD1_SA 18_ID 492_23102016194610.pdf. 2016.

8. In dieser Untersuchungsarbeit, verwendet, um methodische Verweisung zu bezeichnen, die aus der Perspektive der Untersuchung der Konzeptionen, Traditionen und mathematischen Praktiken einer sozialen Gruppe entwickelt werden kann, durch die die Gruppe interpretiert und kodifiziert ihre wissen.

9. Ubiratan D'ambrsio, Professor, mathematischer Pädagoge und Forscher an der Paulista State University (UNESP). Rio Claro Campus. Institut für Geowissenschaften und Exakte Wissenschaften (IGCE). Er ist emeritierter Professor an der Staatlichen Universität Campinas/UNICAMP. Er hat Studien veröffentlicht in: Geschichte und Philosophie der Mathematik, Geschichte und Philosophie der Wissenschaften, Ethnomathematik, Ethnowissenschaften, mathematische Bildung und transdisziplinäre Studien.

10. Der Ethno-Ansatz bezieht sich auf mehrere Perspektiven, die in Bezug auf diesen Bereich im Umlauf sind, der bis heute theoretisch nicht kristallisiert ist. Die pädagogen und Forscher, die an diesem Thema beteiligt waren, beschlossen, Zeit für die 
Explorationen zu geben, um voranzukommen und so zu einer möglichen theoretischen Ausarbeitung der Ethnomathematik beizutragen. Es ist möglich, dass diese Entwicklung im Großen und Ganzen die verschiedenen Perspektiven einschließt, die im Umlauf sind.

11. Dialektik in dieser Reflexion, drückt die Kunst der Suche nach Dialog, durch Argumentation und konterkardiert die Erweiterung der Ideen und Konzepte in die Diskussion beteiligt. Bei der Analyse von Fakten und Situationen-Problem, die die Entwicklung des Denkens ermöglichen, haben Lehrer und Schüler die gleiche dialogische Teilhabe, man hört immer dem anderen zu, (re) baut zusammen ihre Erkenntnisse auf.

Eingereicht: Juni 2019.

Genehmigt: Juli 2019. 